\title{
Structure selection with ANOVA: local linear models
}

\author{
Ingela Lind, Lennart Ljung \\ Division of Automatic Control \\ Department of Electrical Engineering \\ Linköpings universitet, SE-581 83 Linköping, Sweden \\ WWW: http://www. control.isy.liu.se \\ E-mail: ingela@isy.liu.se, ljung@isy.liu.se
}

13th December 2002

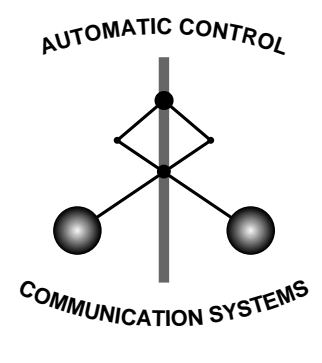

LINKÖPING

Report no.: LiTH-ISY-R-2478

Submitted to SYSID 2003 August, Rotterdam, Netherlands

Technical reports from the Control \& Communication group in Linköping are available at http://www. control.isy.liu.se/publications. 


\begin{abstract}
The structure identification problem when estimating local linear models can be eased by using Analysis of Variance (ANOVA) as a prior step in the estimation procedure. The information gained from using ANOVA on the input/output data is what regressors that should be used to partition the input space and what regressors are needed only for the linear models in each part. Also the complexity of the partitioning can be restricted due to the extra information.
\end{abstract}

Keywords: Identification, Nonlinear models, Analysis of variance 


\title{
STRUCTURE SELECTION WITH ANOVA: LOCAL LINEAR MODELS
}

\author{
Ingela Lind and Lennart Ljung \\ Division of Automatic Control, Department of Electrical Engineering, \\ Linköpings Universitet, SE-581 83 Linköping, Sweden. \\ E-mail: \{ingela, ljung\}@isy.liu.se
}

\begin{abstract}
The structure identification problem when estimating local linear models can be eased by using Analysis of Variance (ANOVA) as a prior step in the estimation procedure. The information gained from using ANOVA on the input/output data is what regressors that should be used to partition the input space and what regressors are needed only for the linear models in each part. Also the complexity of the partitioning can be restricted due to the extra information.
\end{abstract}

Keywords: Identification, Nonlinear models, Analysis of variance

\section{PROBLEM DESCRIPTION}

The problem that will be discussed in this paper is the one of easing the process of building good, parsimonious models of nonlinear systems. The tool used for obtaining this goal is the statistical method analysis of variance (ANOVA). (Miller, 1997) is a comprehensive reference.

In earlier work, it has been shown that ANOVA can be used to extract structure information from input/output data, see (Lind, 2000; Lind, 2001; Lind, 2002). This can be done without estimating any complex model, which is a great benefit due to the complexity of the estimation task. In this contribution it will be shown how the structure information from ANOVA can be applied to the local linear model structure.

\section{THE KEY TO STRUCTURE INFORMATION}

The key to extracting structure information from the data lies in utilising a point model with a special parameterisation, called a linear statistical model (here for a two-dimensional function):

$$
y_{i j k}=\mu+\tau_{i}+\beta_{j}+(\tau \beta)_{i j}+\varepsilon_{i j k},
$$

The overall mean effect is denoted by $\mu . \tau_{i}$ is the mean effect of the $i$ th level of the factor $A$, where $i=1, \ldots, a$, while $\beta_{j}$ is the mean effect of the $j$ th level of the factor $B$, where $j=1, \ldots, b$. The means are taken over all the levels of the other factor. The interactions between the factors are denoted by $(\tau \beta)_{i j}$, which is indexed by the levels of both factors. The random error component $\varepsilon_{i j k}$ is assumed to come from a Gaussian distribution with constant variance $\sigma^{2}$ and the index $k$ denotes the sample number from the $i j$ :th level combination. The number of free parameters in the model is restricted by the following equations, that define all the effects as deviations from lower order interactions (with the overall mean as interaction of order zero): $\sum_{i=1}^{a} \tau_{i}=0, \sum_{j=1}^{b} \beta_{j}=0, \sum_{i=1}^{a}(\tau \beta)_{i j}=$ $0, \forall j$ and $\sum_{j=1}^{b}(\tau \beta)_{i j}=0, \forall i$.

It is possible to divide the residual quadratic sum from this point model into orthogonal parts related to the $\tau_{i}: \mathrm{s}, \beta_{j}: \mathrm{s},(\tau \beta)_{i j}: \mathrm{s}$ and the random error component respectively. These then form $F$-distributed test variables that can be used to determine whether factor $A$ and/or $B$ affect the output at all, additively or with interaction.

For a higher-dimensional point model, the used parameterisation has many more than a sufficient number of parameters. The reason to use this parameterisation is that the high-order interactions are very seldom present in the data, so many of the interaction 
effect parameters can be discarded with the $F$-tests, thereby simplifying the model while giving the wanted structural information.

The linear statistical model structure also benefit the transparency of the model. People tend to think about functional relationships in two- and three-dimensional views and have a hard time understanding complex interactions between many variables. If the model should be useful for physical interpretations the order of the interactions should be kept low. This type of model thinking has been incorporated by (Harris et $a l ., 2002)$ in their SUPANOVA algorithm which is a support vector machine built on the ideas to separate low-order interaction effects from high-order interaction effects by a special function expansion.

\section{LOCAL MODELS}

A local model, as described in (Nelles, 2001; Töpfer $e t$ al., 2002), is a nonlinear model of the following form:

$$
\hat{y}=\sum_{i=1}^{M} \Phi_{i}(\varphi) g_{i}(\varphi),
$$

where a set of local models, the $g_{i}(\varphi): \mathrm{s}$, are weighted by the weighting, activation or membership functions $\Phi_{i}(\varphi)$. The regressor vector $\varphi$ can consist of, e. g., different input time lags, old output lags and/or transformations of these. This model is an alternative function expansion to the one used in neural networks. The idea is that the input space is divided into parts in which a simple model $g_{i}$, most often a linear one, describes the output satisfactory. The $\Phi_{i}$ :s take care of to which part of the input space the input belongs and the possible smoothing of the transitions between the parts. The partitioning of the input space can be done in the following ways; a grid structure, recursive partitioning or a partitioning of arbitrary form. The choice of partitioning is called structure identification. The model can also be seen as a linear model with operating-point dependent parameters;

$$
\begin{aligned}
\hat{y} & =\sum_{i=1}^{M}\left(w_{i 0}+w_{i 1} \varphi_{1}+\ldots w_{i p} \varphi_{p}\right) \Phi_{i}(\varphi)= \\
& =w_{0}(\varphi)+w_{1}(\varphi) \varphi_{1}+\ldots+w_{p}(\varphi) \varphi_{p}
\end{aligned}
$$

with

$$
w_{q}=\sum_{i=1}^{M} w_{q i} \Phi_{i}(\varphi) .
$$

It is not necessarily the case that all regressors in $\varphi$ are included in both $g_{i}(\varphi)$ and $\Phi_{i}(\varphi)$. In the following, the part of $\varphi$ that occurs in $g_{i}$ will be called $\mathbf{x}$ and the part that occurs in $\Phi_{i}$ will be called $\mathbf{z} . \mathbf{x}$ and $\mathbf{z}$ can be partly constituted by the same regressors. As already mentioned the $g_{i}(\mathbf{x})$ are often linear(or affine) functions. Also the notion of cells will be used. Each part of the input space defined in an axis-orthogonal grid is called a cell, see Figure 1.
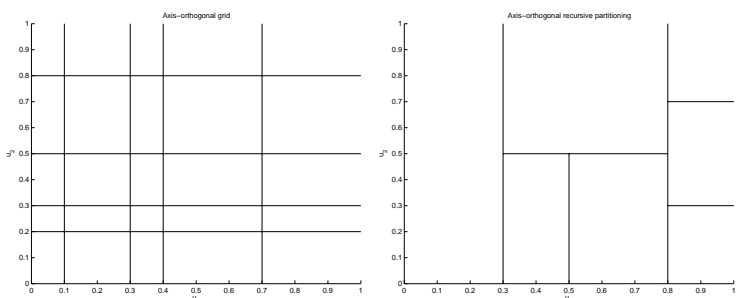

Fig. 1. These are examples of an axis-orthogonal grid to the left and an axis-orthogonal recursive partitioning to the right.

Sometimes the $\Phi_{i}(\mathbf{z})$ are decomposed into functions of the form $f_{i 1}\left(z_{1}\right) f_{i 2}\left(z_{2}\right) \cdot \ldots \cdot f_{i k}\left(z_{k}\right)$, where each $f_{i j}\left(z_{j}\right)$ is a bell shaped function. The spread of the bell depends on whether a smooth transition between different parts of the input space is wanted or not. This decomposition gives an axis-orthogonal partitioning of the input space, see Figure 1.

Here the questions are how many and which $f_{i j}\left(z_{j}\right)$ are needed for successful modelling and which parts of $\varphi$ that should be included in $\mathbf{x}$ and $\mathbf{z}$ respectively. This is exactly the kind of information ANOVA can contribute with. Also process knowledge will be helpful.

\section{REDUCING STRUCTURAL COMPLEXITY}

In this section, data from the system

$$
y=\operatorname{sgn}\left(u_{1}\right) \cdot u_{2}+u_{3}+e
$$

will be used as example. If $u_{1}$ is the derivative of the position, this system is a very simple example in which different dynamics apply when braking or accelerating. Here, $e$ comes from a zero mean normal distribution with variance 1 . The data are collected such that $u_{1}, u_{2}$ and $u_{3}$ each assume the levels $-1,1$, 3 and 5 and all level combinations of these factors are present four times. This gives 256 input/output data. If the factors are different lags of the same signal, the level combinations can be obtained by using a pseudorandom multi-level signal (Godfrey, 1993).

The reduction of the structural complexity can be seen as a three-step procedure:

(1) Apply ANOVA to your data (Lind, 2001). The result is an ANOVA table, see Table 1. From this table, the significant regressors and their interaction pattern can be concluded. In this case, the three-factor interaction is not significant, i. e., all the factors contribute non-additively to the output, and the two-factor interactions between $u_{1}$ and $u_{3}$ and between $u_{2}$ and $u_{3}$ are not significant. The main effect from $u_{3}$ is significant, but it is not interesting to consider the main effects from $u_{1}$ or $u_{2}$ since the interaction between these factors is significant.

(2) The significant effects can be investigated further by looking at plots of the cell means, i. e., the 


\begin{tabular}{l|rrrl} 
Effect & $\begin{array}{r}\text { Degrees of } \\
\text { Freedom }\end{array}$ & $\begin{array}{r}\text { Mean } \\
\text { Square }\end{array}$ & $\mathrm{F}$ & p-level \\
\hline$u_{1}$ & 3 & 248 & 224 & 0.0000 \\
$u_{2}$ & 3 & 111 & 100 & 0.0000 \\
$u_{3}$ & 3 & 427 & 386 & 0.0000 \\
$u_{1} * u_{2}$ & 9 & 111 & 100 & 0.0000 \\
$u_{1} * u_{3}$ & 9 & 0.9 & 0.8 & 0.64 \\
$u_{2} * u_{3}$ & 9 & 1.4 & 1.2 & 0.28 \\
$u_{1} * u_{2} * u_{3}$ & 27 & 1.0 & 1.0 & 0.53 \\
Error & 192 & 1.1 & &
\end{tabular}

Table 1. Analysis of Variance Table. The columns are from the left; the degrees of freedom associated with each sum of squares, the sum of squares divided by its degrees of freedom, the value of the F-distributed test variable associated with the corresponding interaction effect and, finally, the probability level that the interaction is not present.

mean over all the data in each cell, together with their confidence intervals. If a straight line can be drawn through the confidence intervals, in a way that will be explained further, the effect of the factor on the $\mathrm{x}$-axis can be considered to be linear, given the other factor(s) involved in the plot.

(3) Use the information when deciding what possible partitionings to use for estimating the local linear model. See section 4.2.

\subsection{Investigating interaction effects}

To see if an interaction effect is linear in one of the factors given the others, e. g., $y=\operatorname{sgn}\left(u_{1}\right) \cdot u_{2}$ which is linear in $u_{2}$ given $u_{1}$, it is possible to check the plots of the cell means, see Figure 2. These plots are computationally "free" and they also give an indication of how reliable the linear assumption is. No extra computations are needed for the plots of cell means, since all the cell means are computed in order to make the ANOVA table. A good estimate of the error variance is also obtained, so confidence intervals for the cell means are easily computed. The linearity tests done by looking at these plots can be done formally by defining linear contrasts, which is a linear combination of the cell means nominally adding to zero. A confidence interval for the linear contrast will tell if the difference from zero is significant (no linearity) or not.

It is enough to look at the cell mean plots for the highest order interaction of significance (for each factor combination). Assume that the cell means (in a twodimensional grid) are linear functions of factor B for each fixed level $i$ of factor $\mathrm{A}$, that is,

$$
\mu_{i j}=\mu+\tau_{i}+\beta_{j}+(\tau \beta)_{i j}=v_{i}+\rho_{i} \delta_{j},
$$

where $\mu_{i j}$ is the cell mean in cell $(i, j), v_{i}$ is a constant offset, $\rho_{i}$ is the slope and $\delta_{j}$ is the distance between level 1 and level $j$ of factor $\mathrm{B}$. The row means $\mu_{j}$ are computed as

$$
\begin{aligned}
\mu_{j} & =\frac{1}{a} \sum_{i=1}^{a} \mu_{i j}=\mu+\beta_{j}= \\
& =\frac{1}{a} \sum_{i=1}^{a} v_{i}+\left(\frac{1}{a} \sum_{i=1}^{a} \rho_{i}\right) \delta_{j}=v+\rho \delta_{j},
\end{aligned}
$$

where the second equality is due to the parameter restrictions and $v=\frac{1}{a} \sum_{i=1}^{a} v_{i}$ and $\rho=\frac{1}{a} \sum_{i=1}^{a} \rho_{i}$. Hence, it is obvious that if the two-dimensional cell means show a linear relation for factor $\mathrm{B}$, also the onedimensional cell means (the row means) will show a linear relation.

If high-order interaction effects are present, as shown by the ANOVA table, there will be more plots to look at. The axes of the high-dimensional cell means plot have to be permuted such that each regressor in the significant interaction effect gets to be at the $\mathrm{x}$ axis once. Then the regressors that only need to be included in $\mathbf{x}$, the regressor vector for the local linear models, will be detected. The number of plots for each permutation will vary depending on how many levels each factor has and the order of the significant interaction.

\subsection{Corresponding local linear model structure}

Listed below are the possible outcomes of steps 1 and 2 and the corresponding choices of $\mathbf{x}, \mathbf{z}$ and $\Phi_{i}(\mathbf{z})$, for an analysis with three factors. How many $\Phi_{i}(\mathbf{z})$ :s that are needed cannot be concluded from these tests. The idea is quite simple, but there are many slightly different cases, hence the length of the list.

1a; Three-factor interaction between $u_{1}, u_{2}$ and $u_{3}$. No linearities detected in the cell means plots. Let $\mathbf{x}=\left[u_{1}, u_{2}, u_{3}\right]$ and $\mathbf{z}=\left[u_{1}, u_{2}, u_{3}\right]$. Possible $\Phi_{i}(\mathbf{z})$ :s are $f_{i 1}\left(u_{1}\right) \cdot f_{i 2}\left(u_{2}\right) \cdot f_{i 3}\left(u_{3}\right)$.

$1 \mathrm{~b}$; Three-factor interaction between $u_{1}, u_{2}$ and $u_{3}$. Linear in $u_{1}$ given the levels of $u_{2}$ and $u_{3}$. Let $\mathbf{x}=\left[u_{1}, u_{2}, u_{3}\right]$ and $\mathbf{z}=\left[u_{2}, u_{3}\right]$. Possible $\Phi_{i}(\mathbf{z}): \mathrm{s}$ are $f_{i 2}\left(u_{2}\right) \cdot f_{i 3}\left(u_{3}\right)$.

$1 \mathrm{c}$; Analogue to case $1 \mathrm{~b}$, but with $u_{2}$ linear given the others.

$1 \mathrm{~d}$; Analogue to case $1 \mathrm{~b}$, but with $u_{3}$ linear given the others.

1e; Three-factor interaction between $u_{1}, u_{2}$ and $u_{3}$. Linear in $u_{1}$ given the levels of $u_{2}$ and $u_{3}$, and in $u_{2}$ given the levels of $u_{1}$ and $u_{3}$. Choose between using the setup in case $1 \mathrm{~b}\left(u_{2}\right.$ as regime variable) or the setup in case $1 \mathrm{c}\left(u_{1}\right.$ as regime variable).

1f; Analogue to case 1e, but with $u_{1}$ or $u_{3}$ linear given the others.

$1 \mathrm{~g}$; Analogue to case $1 \mathrm{e}$, but with $u_{2}$ or $u_{3}$ linear given the others.

1h; Three-factor interaction between $u_{1}, u_{2}$ and $u_{3}$. Linear in $u_{1}$ given the levels of $u_{2}$ and $u_{3}$, in $u_{2}$ given the levels of $u_{1}$ and $u_{3}$, and in $u_{3}$ given the levels of $u_{1}$ and $u_{2}$. Choose between using the setup in case $1 \mathrm{~b}\left(u_{2}\right.$ and $u_{3}$ as regime variables), the setup in case $1 \mathrm{c}\left(u_{1}\right.$ and $u_{2}$ as 

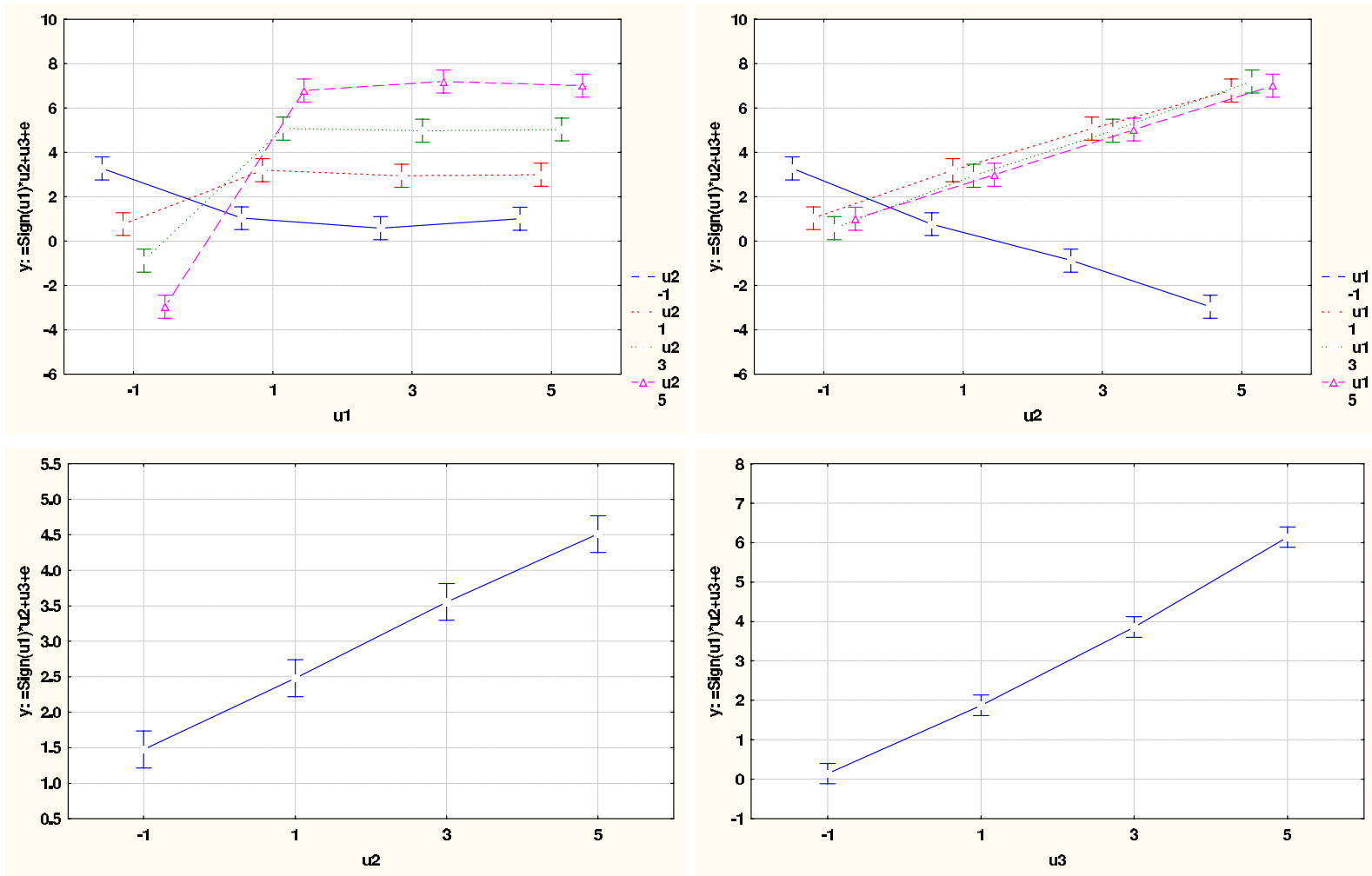

Fig. 2. Cell mean plots with confidence intervals. The upper two plots show the two-dimensional cell means, corresponding to the interaction between $u_{1}$ and $u_{2}$. The plot to the left have $u_{1}$ on the $\mathrm{x}$-axis and one line for each value of $u_{2}$, while the plot to the right have $u_{2}$ on the x-axis. From the plots, the following conclusions can be drawn: $u_{1}$ and $u_{2}$ affect $y$ with interaction, since the curves within each plot do not have the same shape. $u_{2}$ affects $y$ linearly if the value of $u_{1}$ is fixed, since it is possible to draw a straight line through the confidence intervals for $\mu_{i j}$ for each value of $i$. (The lines in the plot only connects the cell means, they are not straight lines). The lower plots show the one-dimensional cell means with $u_{2}$ and $u_{3}$ on the $\mathrm{x}$-axis respectively. The left plot confirms that the important information is present in the two-dimensional cell means plots. The right plot shows that the effect from $u_{3}$ could be linear.

regime variables), or the setup in case $1 \mathrm{~d}\left(u_{1}\right.$ and $u_{3}$ as regime variables).

2; Two-factor interactions between $u_{1}$ and $u_{2}$, between $u_{1}$ and $u_{3}$ and between $u_{2}$ and $u_{3}$ but no three-factor interaction. The model can be decomposed into three additive sub-models. See further cases 5.1, 5.2 and 5.3. When there are possible choices of which variables should act as regime variables it could be wise to consider the possible choices in the other sub-models too.

3.1; Two-factor interactions between $u_{1}$ and $u_{2}$, and between $u_{1}$ and $u_{3}$. The model can be decomposed into two additive sub-models. See further cases 5.1 and 5.3.

3.2; Two-factor interactions between $u_{1}$ and $u_{3}$, and between $u_{2}$ and $u_{3}$. The model can be decomposed into two additive sub-models. See further cases 5.1 and 5.2.

3.3; Two-factor interactions between $u_{1}$ and $u_{2}$, and between $u_{2}$ and $u_{3}$. The model can be decomposed into two additive sub-models. See further cases 5.2 and 5.3.

4.1; Two-factor interaction between $u_{1}$ and $u_{2}$ and main effect from $u_{3}$. The model can be decom- posed into two additive sub-models. See further cases 5.1 and 7.3.

4.2; Two-factor interaction between $u_{1}$ and $u_{3}$ and main effect from $u_{2}$. The model can be decomposed into two additive sub-models. See further cases 5.2 and 7.2 .

4.3; Two-factor interaction between $u_{2}$ and $u_{3}$ and main effect from $u_{1}$. The model can be decomposed into two additive sub-models. See further cases 5.3 and 7.1.

5.1a; Two-factor interaction between $u_{1}$ and $u_{2}$. No linearities. Let $\mathbf{x}=\left[u_{1}, u_{2}\right]$ and $\mathbf{z}=\left[u_{1}, u_{2}\right]$. Possible $\Phi_{i}(\mathbf{z})$ :s are $f_{i 1}\left(u_{1}\right) \cdot f_{i 2}\left(u_{2}\right)$.

$5.1 \mathrm{~b}$; Two-factor interaction between $u_{1}$ and $u_{2}$. Linear in $u_{1}$ given the levels of $u_{2}$. Let $\mathbf{x}=\left[u_{1}, u_{2}\right]$ and $\mathbf{z}=\left[u_{2}\right]$. Possible $\Phi_{i}(\mathbf{z})$ :s are $f_{i 2}\left(u_{2}\right)$.

5.1c; Two-factor interaction between $u_{1}$ and $u_{2}$. Linear in $u_{2}$ given the levels of $u_{1}$. Let $\mathbf{x}=\left[u_{1}, u_{2}\right]$ and $\mathbf{z}=\left[u_{1}\right]$. Possible $\Phi_{i}(\mathbf{z})$ :s are $f_{i 1}\left(u_{1}\right)$.

5.1d; Two-factor interaction between $u_{1}$ and $u_{2}$. Linear in $u_{1}$ given the levels of $u_{2}$ and linear in $u_{2}$ given the levels of $u_{1}$. Choose between the setup in case $5.1 \mathrm{~b}\left(u_{2}\right.$ as regime variable $)$, and the setup in case 5.1c ( $u_{1}$ as regime variable). 
5.2; Two-factor interaction between $u_{1}$ and $u_{3}$. Analogue to case 5.1.

5.3; Two-factor interaction between $u_{2}$ and $u_{3}$. Analogue to case 5.1.

6.1; Main effects from $u_{1}$ and $u_{2}$. No interactions. The model can be decomposed into two additive sub-models. See further cases 7.1 and 7.2.

6.2; Main effects from $u_{1}$ and $u_{3}$. The model can be decomposed into two additive sub-models. See further cases 7.1 and 7.3.

6.3; Main effects from $u_{2}$ and $u_{3}$. The model can be decomposed into two additive sub-models. See further cases 7.1 and 7.2.

7.1a; Main effect from $u_{1} \cdot u_{1}$ is linear. Let $\mathbf{x}=\left[u_{1}\right]$ and $\mathbf{z}$ empty. No partitioning is needed.

7.1b; Main effect from $u_{1} \cdot u_{1}$ is nonlinear. Let $\mathbf{x}=\left[u_{1}\right]$ and $\mathbf{z}=\left[u_{1}\right]$. Possible $\Phi_{i}(\mathbf{z})$ :s are $f_{i 1}\left(u_{1}\right)$.

7.2; Main effect from $u_{2}$. Analogue to case 7.1.

7.3; Main effect from $u_{3}$. Analogue to case 7.1.

8; No significant effects. None of the tested factors show systematic effects in the data.

From the example data set, we have gained the information that all the factors $u_{1}, u_{2}$ and $u_{3}$ are present in the model. The model structure according to the ANOVA table is

$$
y=h_{1}\left(u_{1}, u_{2}\right)+h_{2}\left(u_{3}\right)+e,
$$

which is case 4.1 above. The interaction between $u_{1}$ and $u_{2}$ was then investigated further (Figure 2), giving the result that $h_{1}\left(u_{1}, u_{2}\right)=f\left(u_{1}\right) \cdot u_{2}$, that is, $u_{1}$ belongs to the regime variables $\mathbf{z}$ while $u_{2}$ belongs to $\mathbf{x}$. If $h_{2}\left(u_{3}\right)$ is a nonlinear function, $u_{3}$ need to be in both $\mathbf{z}$ and $\mathbf{x}$. Since $u_{1}$ and $u_{3}$ do not interact the weighting functions $\Phi_{i}$ will be either $f_{i 1}\left(u_{1}\right)$ or $f_{i 3}\left(u_{3}\right)$, but not $f_{i 1}\left(u_{1}\right) \cdot f_{i 3}\left(u_{3}\right)$. The linearity can be tested by plotting the cell means against the levels of factor $\mathrm{C}\left(u_{3}\right)$, see Figure 2, which shows that $h_{2}\left(u_{3}\right)$ could be linear and $u_{3}$ does not need to be included in z. The total model consist of two additive sub-models, one corresponding to case $5.1 \mathrm{c}$ and the other to case 7.3a.

\subsection{Another example}

The second example is a bit more complicated than the first one;

$$
y=\operatorname{sgn}\left(u_{1}\right) \cdot u_{2} \cdot u_{3}+u_{3}^{2}+e .
$$

The inputs and the noise are chosen exactly as in the first example and the amount of data is the same. The ANOVA table is given in Table 2 and shows that we have case 1 in the list in Section 4.2. The linearity is investigated in the cell mean plots, see Figures 3, 4 and 5 . These show that we have case 1c. That means that we should choose $\mathbf{x}$ as $\left[u_{1}, u_{2}, u_{3}\right]$ and $\mathbf{z}$ as $\left[u_{2}, u_{3}\right]$. Possible $\Phi_{i}(\mathbf{z})$ :s are $f_{i 2}\left(u_{2}\right) \cdot f_{i 3}\left(u_{3}\right)$, but exactly where the partitionings should go is an identification issue.

\begin{tabular}{l|rrrr} 
Effect & $\begin{array}{r}\text { Degrees of } \\
\text { Freedom }\end{array}$ & $\begin{array}{r}\text { Mean } \\
\text { Square }\end{array}$ & $\mathrm{F}$ & p-level \\
\hline$u_{1}$ & 3 & 1008 & 910 & 0.0000 \\
$u_{2}$ & 3 & 435 & 392 & 0.0000 \\
$u_{3}$ & 3 & 12122 & 10941 & 0.0000 \\
$u_{1} * u_{2}$ & 9 & 435 & 392 & 0.0000 \\
$u_{1} * u_{3}$ & 9 & 423 & 382 & 0.0000 \\
$u_{2} * u_{3}$ & 9 & 183 & 165 & 0.0000 \\
$u_{1} * u_{2} * u_{3}$ & 127 & 170 & 154 & 0.0000 \\
Error & 192 & 1.1 & & \\
\hline
\end{tabular}

Table 2. Analysis of Variance Table for the second example.

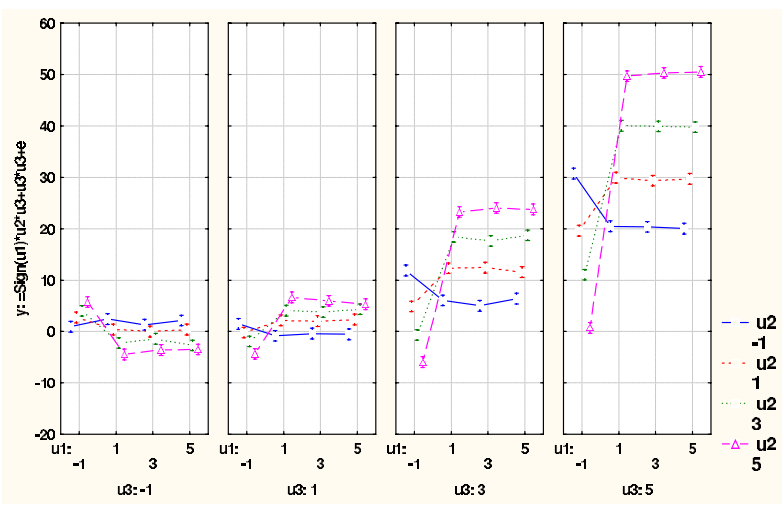

Fig. 3. Cell mean plots with confidence intervals. $u_{1}$ on the $\mathrm{x}$-axis.

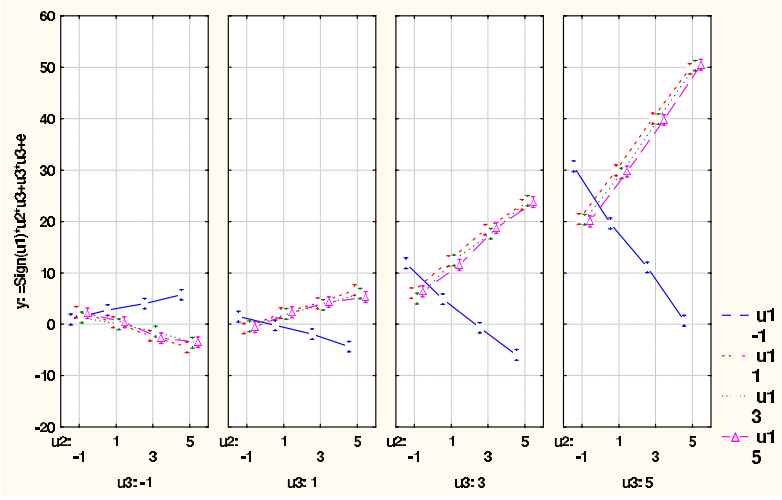

Fig. 4. Cell mean plots with confidence intervals. $u_{2}$ on the $\mathrm{x}$-axis. The effects seem linear.

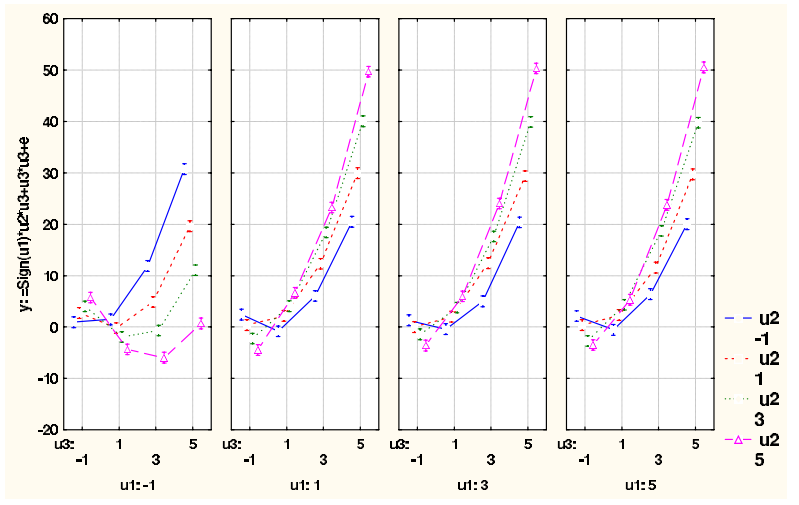

Fig. 5. Cell mean plots with confidence intervals. $u_{3}$ on the $\mathrm{x}$-axis. 


\section{CONCLUSIONS}

It is possible to gain enough structure information from the input/output data to assist the structure identification task in local linear modelling by using ANOVA. The information that can be extracted from the ANOVA table and the cell means plots are what regressors that should be used for the linear models in each part of the input space, what regressors that should determine the partitioning of the input space and how complex the partitioning should be (that is, how many regressors interact in each weighting function). The cell means plots with confidence intervals appeal also to intuition. They are very similar to scatter plots, but with the benefit that the statistical properties of the input/output data are made visible. Left to consider in the structure identification task is how many partitionings to use and where the limits of each part should be.

\section{ACKNOWLEDGEMENTS}

This work has been supported by the Swedish Research Council (VR), which is gratefully acknowledged.

\section{REFERENCES}

Godfrey, Keith (1993). Perturbation Signals for System Identification. Prentice Hall. New York.

Harris, C., X. Hong and Q. Gan (2002). Adaptive Modelling, Estimation and Fusion from Data: a neurofuzzy approach. Springer-Verlag. Berlin Heidelberg.

Lind, Ingela (2000). Model order selection of N-FIR models by the analysis of variance method. In: Proc IFAC Symposium SYSID 2000. Santa Barbara. pp. We PM4-4.

Lind, Ingela (2001). Regressor selection in system identification using ANOVA. Technical Report Licentiate Thesis no. 921. Department of Electrical Engineering, Linköping University. SE-581 83 Linköping, Sweden.

Lind, Ingela (2002). Regressor selection with the analysis of variance method. In: Proc 15th IFAC World Congress, 21-26 July, 2002. Barcelona, Spain. pp. T-Th-E 012.

Miller, Jr., Rupert G. (1997). Beyond ANOVA. Chapman and Hall. London.

Nelles, O. (2001). Nonlinear System Identification. Springer-Verlag. Berlin Heidelberg.

Töpfer, S., A. Wolfram and R. Isermann (2002). Semiphysical modelling of nonlinear processes by means of local approaches. In: Proc 15th IFAC World Congress, 21-26 July, 2002. Barcelona, Spain. pp. T-Th-M 015. 